Risk factors for recurrence of febrile seizures were reported in a study of FS in Southern Chinese children (Chung BC et al. Pediatr Neurol 2006;34:121-126). Factors reaching statistical significance included: 1) earlier age of onset $<1.5$ years; 2) family history of FS; 3 ) CFS as first FS; and 4) relatively lower degree of fever (low threshold) with first FS in patients with recurrence vs those with no recurrence. Factors not significant in determining risk of recurrence of FS included sex, family history of epilepsy, and duration of fever during the FS illness. This study confirms the long established theory of height of the fever as a measure of the FS threshold and risk factor for recurrent FS and epilepsy. (See Ped Neur Briefs May 1995 and May 1994).

\title{
LATERALIZING SIGNS IN PARTIAL EPILEPSY
}

Lateralizing signs (LSs) in 100 children of 12 or $<12$ years of age with localizationrelated epilepsy were studied while undergoing presurgical evaluation at the Epilepsy Center Bethel, Bielefeld, Germany. Seizures were localized by video-EEG recordings and cortical resections and involved the frontal lobe in $21 \%$ patients, temporal lobe in $61 \%$, occipital lobe in $12 \%$, and parieto-occipital in $6 \%$. The side of seizure onset was defined by seizure freedom after cortical resection. Etiology of epilepsy was a cortical malformation in 49, dysgenetic tumor in 32 , hippocampal sclerosis in 9 , and various pathologies, including tuberous sclerosis, stroke, Sturge-Weber syndrome, and abscess, in 10. LSs occurred in $75 \%$ children and were more frequent in older children $(p=0.001)$. The following LSs were significantly more common in older children (manual automatism, dystonic posturing, version, postictal dysphasia, and postictal facial wiping), whereas the following showed no significant differences with age (postictal nose wiping, unilateral tonic or clonic seizures, Todd's paralysis, and nystagmus). The sum of different LSs showed a linear relation with age at monitoring $(\mathrm{p}<0.001)$. (Fogarasi A, Janszky J, Tuxhorn I. Peri-ictal lateralizing signs in children: blinded multiobserver study of 100 children $<12$ years. Neurology January ( 2 of 2$)$ 2006;66:271-274). (Reprints: Dr Andras Fogarasi, Epilepsy Center, Bethesda Children's Hospital, Bethesda Street 3, H-1146-Budapest, Hungary).

COMMENT. LSs are less frequent in children than in adults but are of high localizing value. They are more common in older than in younger children, and their development appears to correlate with brain maturation.

Partial seizures in progressive multifocal leukoencephalopathy (PML). Review of records of 89 patients (age range 14-81 years; mean age 44.8 years) with PML showed a seizure prevalence of $18 \%$, and much higher than expected in a white matter disease. Seven (44\%) had simple partial seizures, $2(12 \%)$ complex partial seizures, and $7(14 \%)$ partial with secondary generalization. MRI showed lesions adjacent to hemispheric cortex in 15 (94\%) of 16 PML patients with seizures. Eighty percent of patients with PML have HIV infection or other immunosuppressive illness. New-onset partial seizures in immunosuppressed patients should signal tests for PML (Lima MA et al. Neurology Jan (2 of 2) 2006;66:262-264). 